Recherches en histoire de l'art, histoire des civilisations, archéologie, anthropologie et muséologie

$15 \mid 2020$

Cahier $\mathrm{n}^{\circ} 15$

\title{
Des reines violentes
}

Le genre et la guerre dans l'iconographie royale française au XVII ${ }^{\mathrm{e}}$ siècle Violent queens. Gender and war in French royal iconography in the 17th century

Damien Bril

\section{(2) OpenEdition}

Journals

Édition électronique

URL : http://journals.openedition.org/cel/9736

DOI : $10.4000 /$ cel.9736

ISSN : 2262-208X

Éditeur

École du Louvre

Référence électronique

Damien Bril, «Des reines violentes », Les Cahiers de l'École du Louvre [En ligne], 15 | 2020, mis en ligne le 03 novembre 2020, consulté le 05 novembre 2020. URL : http://journals.openedition.org/cel/9736 ;

DOI : https://doi.org/10.4000/cel.9736

Ce document a été généré automatiquement le 5 novembre 2020.

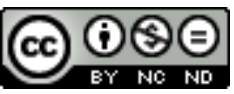

Les Cahiers de l'École du Louvre sont mis à disposition selon les termes de la licence Creative Commons Attribution - Pas d'Utilisation Commerciale - Pas de Modification 4.0 International. 


\section{Des reines violentes}

Le genre et la guerre dans l'iconographie royale française au XVII siècle

Violent queens. Gender and war in French royal iconography in the 17th century

\section{Damien Bril}

1 Les représentations du prince cristallisent les tensions qui traversent les sociétés d'Ancien Régime dans leur rapport à la violence. L'arrivée sur le trône de la dynastie Bourbon à la fin du XVI siècle correspond à une transformation de la culture de la violence politique : à la lecture eschatologique d'un « roi violent » succède celle d'un roi guerrier gouvernant ses sujets comme Dieu son royaume ${ }^{1}$. Les supports visuels participent activement à cette affirmation d'un « roi de guerre », en armes et sur le champ de bataille, qui prend une place de plus en plus importante parmi les portraits du souverain au cours du XVII ${ }^{e}$ siècle ${ }^{2}$. Des scènes de batailles conduites par Henri IV déclinent ainsi l'image d'un roi à la tête de ses troupes, donnant la charge l'épée à la main, comme sur la bataille d'Arques attribué à Jacob Bunel, emblématique d'une production contemporaine que l'estampe diffuse largement ${ }^{3}$. Quarante ans plus tard, Louis XIII est encore représenté au cœur de la geste guerrièré, alors même que les progrès de l'artillerie et l'évolution de l'administration de la guerre rendent moins utile et plus dangereuse sa présence personnelle sur le front.

2 Le développement de cette iconographie au cours du premier XVII ${ }^{e}$ siècle repose sur son efficacité allégorique et le thème ne traverse d'ailleurs pas seulement les images narratives mais également les figurations symboliques : Henri IV affrontant l'Hydre de Lerne devient par exemple l'évocation d'un souverain triomphant des divisions internes ${ }^{5}$. La violence apparaît ainsi non seulement comme l'instrument de défense du royaume mais aussi comme la métaphore d'une pacification sociale dont le roi est l'agent. Cette concentration symbolique de la force de la monarchie dans les mains de son dirigeant dans les représentations visuelles semble faire écho au courant historiographique qui, depuis Max Weber ${ }^{6}$ et Norbert Elias ${ }^{7}$, esquisse l'histoire de l'État moderne comme l'affermissement progressif d'un monopole de la violence légitime, même si des travaux récents ont permis une réévaluation sensible de cette hypothèse ${ }^{8}$. 
3 La participation des reines à ce processus iconographique est plus complexe à mesurer et à interpréter. Deux critères la conditionnent. En premier lieu, la reine est en France une coquille vide de pouvoir, une ombre qui partage avec le roi la dignité souveraine, mais pas la réalité du pouvoir, son exercice effectif ${ }^{9}$. La reine ne peut pas véritablement exercer une violence, car elle ne maîtrise pas les moyens coercitifs - militaires notamment - dont son mari a la charge au nom de l'État. Rapportée à la condition du genre et aux limitations - juridiques, symboliques et supposées « naturelles » - qu'elles déterminent dans la société, la violence féminine est en outre largement dévalorisée par la culture contemporaine. Le rapport de la femme à la violence est plus souvent celui de la victime, dans les témoignages des sources judiciaires comme dans la culture littéraire ou artistique, et lorsque la femme apparait violente, c'est le signe d'un « retournement » des valeurs du monde, comme l'illustrent des estampes populaires ${ }^{10}$ ou certaines planches satiriques d'Abraham Bosse ${ }^{11}$, où l'épouse effraie et bat son mari $^{12}$. Seule la hiérarchie des ordres sociaux autorise la transgression de ces normes: la noblesse d'une femme lui permet de prendre les armes et de devenir combattante ${ }^{13}$.

\section{Violences historiques : les galeries royales de femmes fortes}

4 Dans le domaine visuel, le thème des «femmes fortes » offre une image sensible à ce paradoxe. La mode de la représentation de galerie de dames illustres propose non seulement l'actualisation d'une modalité de rapport à l'histoire par les grandes figures - déjà développée au Moyen Âge sous la forme du thème des Neuf Preuses en pendant au Neuf Preux - mais nourrit également la "querelle des femmes » contemporaine en illustrant, par l'exemple, la bravoure de femmes du passé présentées comme des modèles vertueux : des héroïnes ${ }^{14}$. Le thème apparaît d'ailleurs comme un moyen pour les reines de suggérer la dimension guerrière de leur identité, en « intensifiant ${ }^{15}$ » leur présence physique dans l'environnement habituel de la résidence où elles vivent, reçoivent et agissent. Marie de Médicis inscrit ainsi des scènes tirées de sa vie et de celle de Catherine de Médicis dans le cadre d'une glorification dynastique: dans les années 1620 , elle commande à plusieurs artistes florentins une série de dix toiles pour présenter les hauts faits des Médicis, afin d'orner les murs du cabinet doré de son appartement au palais du Luxembourg ${ }^{16}$. L'une des œuvres représente Troilo Orsini, envoyé par le grand-duc de Toscane Cosme $\mathrm{I}^{\mathrm{er}}$ de Médicis pour venir apporter son aide à Charles IX dans la lutte contre les Huguenots. La mère du jeune roi de France, Catherine de Médicis, apparaît en position éminente, intermédiaire diplomatique et politique entre la Florence des Médicis et le roi de France. La rencontre n'est toutefois pas dépeinte dans l'environnement feutré d'un cabinet mais projetée en plein cœur d'un espace militaire ; autour de la reine mère, qui domine dans son grand habit noir toute la composition, Charles IX et Troilo Orsini portent l'armure et précèdent de vastes troupes qui s'amassent à l'arrière-plan.

5 Au cours de la régence de Louis XIV, Anne d'Autriche adopte elle aussi le thème des femmes fortes: une suite de portraits féminins est commandée pour décorer son appartement au Palais-Royal, au début de la régence ${ }^{17}$, et une décennie plus tard elle fait orner la chambre de son appartement d'été au Louvre d'une représentation de Judith sur le point de décapiter Holopherne, au-dessus de la porte d'entrée ${ }^{18}$. La violence contenue et anticipée dans le geste de l'héroïne biblique n'était pas 
contradictoire avec le luxe du décor de la pièce ni avec sa fonction : elle répondait à cette culture visuelle des femmes fortes, répandue dans les intérieurs aristocratiques contemporains et justifiant - sinon légitimant - la prise en main des actions politiques par des femmes pendant cette période de régence féminine. Judith, veuve pieuse qui s'était illustrée par les armes, suggérait un parallèle avec la reine mère alors sortie victorieuse de l'épisode de la Fronde, comme un ultime avertissement s'adressant à tout visiteur quittant sa chambre.

6 L'évocation n'était cependant pas l'unique instrument d'affirmation active d'une violence féminine. Plusieurs contemporaines étaient parvenues à intégrer leur portrait personnel dans la représentation de la guerre, dans des situations où la poursuite de la politique par d'autres moyens justifiait la transgression - néanmoins exceptionnelle des interdits du genre dans le maniement des armes. Parmi quelques exemples célèbres, Madame de Saint-Balmont avait non seulement endossé les responsabilités viriles de la défense de son domaine, mais également participé personnellement à la promotion de son image guerrière auprès de la régente Anne d'Autriche, à laquelle elle avait envoyé son portrait dans un contexte militaire ${ }^{19}$.

7 Quoiqu'elles n'aient jamais tenu elles-mêmes les armes, Marie de Médicis comme Anne d'Autriche, qui s'engagèrent toutes deux dans la conduite du gouvernement à la faveur d'une période de régence, mobilisèrent également plus que le simple langage de la suggestion et mirent en scène dans leur portrait personnel la violence de l'État comme image de leur pouvoir. Pour cela, elles ne firent toutefois pas appel aux mêmes moyens iconographiques, ni n'obtinrent les mêmes effets.

\section{Marie de Médicis ou la violence mythologique}

Chez Marie de Médicis, le thème guerrier s'affirme à travers le travestissement du portrait mythologique, qui apparaît très tôt dans le règne ${ }^{20}$. Dès 1603 , une médaille de Guillaume Dupré présente la reine en Minerve face à Henri IV en Mars, de part et d'autre du jeune dauphin ${ }^{21}$. La référence à la déesse de la sagesse, de la stratégie et de la guerre permet de tempérer la violence qu'incarne le roi et de suggérer la réponse que la paix apporte à la guerre, mais les attributs de la déesse - casque et bouclier - sont clairement tirés de l'attirail du soldat et symbolisent la force contraignante de la puissance militaire. Toutefois, le thème ne reparaît pas lorsque la reine est régente, au nom du jeune Louis XIII de 1610 à 1614, et c'est en réalité surtout lorsqu'elle atteint le statut de reine mère ou reine douairière en septembre 1614 que se développe véritablement cette iconographie. À cette position « légale » fixe correspondent en fait plusieurs positions "politiques» en raison des relations difficiles avec le jeune roi : écartée du Conseil en 1617, elle ne le réintègre qu'en 1620, et ses rapports instables avec Louis XIII conduiront finalement à son exil définitif, après $1630^{22}$.

Dans les années 1620 , forte de sa position et de sa confiance retrouvées, elle poursuit l'aménagement du palais du Luxembourg, sa nouvelle résidence très fastueuse aux portes de Paris, dont le décor se veut une affirmation de son autorité23. Au long du cycle de peintures que Rubens consacre à sa vie pour illustrer la galerie de son appartement, et qui multiplie son image personnelle, le thème principal devait être la glorification d'une reine de paix. L'image qui ouvre la galerie, au-dessus de la cheminée, est un grand portrait en pied qui représente, selon une source contemporaine conservée parmi les manuscrits Baluze, «la reine triomphante ${ }^{24}$ ». Les attributs qu'elle tient, 
notamment la Victoire de la main droite, et le casque orné d'un sphinx, désignent bien la Minerve Victorieuse, divinité tutélaire et emblématique de la reine. Toutefois, comme l'a récemment souligné Marianne Cojannot-Le Blanc, la source manuscrite en question, ne fournit pas une liste définitive des peintures, mais donne plutôt un état des sujets retenus, dans les premiers temps du chantier, et il ne faut donc pas surestimer sa portée dans l'interprétation du tableau ${ }^{25}$. De fait, dès la deuxième moitié $\mathrm{du}$ XVII e siècle, les auteurs qui s'attachent à décrire les peintures du cycle y voient moins la référence au triomphe que l'évocation d'un portrait historié: une représentation en Minerve, voire en Bellone, la déesse de la guerre, que désignent ses attributs guerriers qui occupent une place importante dans la composition ${ }^{26}$. L'ambiguïté de lecture est en soi signifiante : la paix ne fait pas disparaître la menace de la violence - elle ne peut que la contenir. Au regard de l'esquisse réalisée pour la peinture, Rubens a d'ailleurs accentué dans la toile finale la présence menaçante du canon qui pointe en oblique, réponse symétrique au bras de la reine: si Marie de Médicis est bien une reine de paix, c'est une paix conçue non pas comme un état stable de quiétude mais comme un équilibre en tension, une action de la force royale qui vient s'opposer aux armes par les armes.

Fig. 1

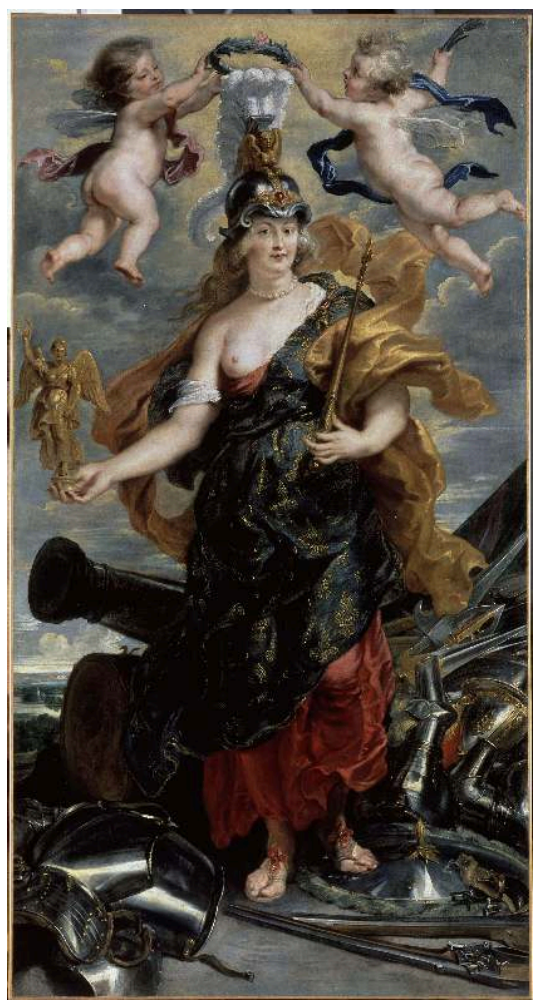

Pierre Paul Rubens, Portrait de Marie de Médicis en Bellone, 1621-1625, huile sur toile, Paris. (c) RMN-Grand Palais (musée du Louvre) / Jean Schormans

Dans le même cycle, Le Triomphe de Juliers repose sur la même appropriation par la reine des signes de l'autorité par les armes. Le thème principal en est encore la paix, mais celle-ci est représentée sous la forme d'un rapport de forces résolu par un conflit militaire. Sur le plan politique et stratégique, ce n'est qu'une petite victoire, sur laquelle les Français ont eu une action certes décisive mais tardive, et assez limitée, en 
renfort de troupes hollandaises. La mise en scène glorieuse donne pourtant à la reine l'occasion d'endosser une iconographie habituellement réservée au roi de guerre : elle figure à cheval, tenant le bâton de commandement, qui permet d'identifier le souverain sur le champ de bataille. Le spectateur de l'époque perçoit probablement sans difficulté l'allusion au monument équestre d'Henri IV que Marie de Médicis avait précisément fait ériger à Paris une décennie plus tôt, en 1614, sur le Pont-Neuf ${ }^{27}$. Bien qu'elle n'ait pas d'armure à la différence du roi, la reine porte un casque empanaché très imposant par-dessus lequel une Gloire la couronne, une manière de revendiquer une action militaire effectuée au tout début de la régence, à l'été 1610, qui apparait donc comme inscrite dans la continuité de l'action royale de son époux défunt, au détriment d'ailleurs du jeune roi Louis XIII, qui demeure ici absent ${ }^{28}$.

Fig. 2



Pierre Paul Rubens, Le Triomphe de Juliers (Dit autrefois : le voyage de Marie de Médicis au Pont-de-Cé en Anjou) le $1^{\text {er }}$ septembre 1610, 1621-1625, huile sur toile, Paris.

(c) RMN-Grand Palais (musée du Louvre) / René-Gabriel Ojéda / Thierry Le Mage

11 L'accessibilité difficile de ces œuvres oppose toutefois de sérieuses limites à la participation de Marie de Médicis à la constitution d'une iconographie féminine guerrière. Accessibilité physique d'abord, puisque, par-delà la présentation officielle du décor dans le cadre du mariage de la sœur du roi Henriette-Marie en 1625, la galerie, comme Sara Galletti l'a récemment rappelé et démontré, n'était pas ouverte largement au public, mais constituait un espace réservé dans l'appartement de la reine, visitable à sa seule discrétion ${ }^{29}$. Accessibilité symbolique également, puisque le maniement très complexe des attributs allégoriques et des figures mythologiques par Rubens en rendait le déchiffrage particulièrement difficile, même pour les contemporains. 


\section{Partager la gloire des armes : un roi de guerre, une reine de paix}

Les effets de cette attention à la dimension militaire de l'image de la reine mère s'observent dans les mêmes années sur l'image de la nouvelle reine régnante, Anne d'Autriche, alors que celle-ci, à la différence de Marie de Médicis, n'exerce pourtant aucun pouvoir et se voit limiter à un rôle de représentation. Parmi les estampes qui sont gravées à partir de la signature du contrat de mariage en 1612, et diffusées en grand nombre jusqu'à l'arrivée de la fille du roi d'Espagne en France en 1615, une planche met en scène l'iconographie du triomphe de l'empereur ${ }^{30}$. L'image reflète assez précisément un type de composition bien connue par plusieurs témoignages antiques ${ }^{31}$ et renforce donc la portée politique de l'alliance entre les couronnes de France et d'Espagne. Conçue en principe au bénéfice de Marie de Médicis, véritable instigatrice du mariage, l'œuvre profite aussi sur le plan visuel à la jeune reine épousée, qui est pleinement intégrée à la gloire militaire du roi.

Fig. 3

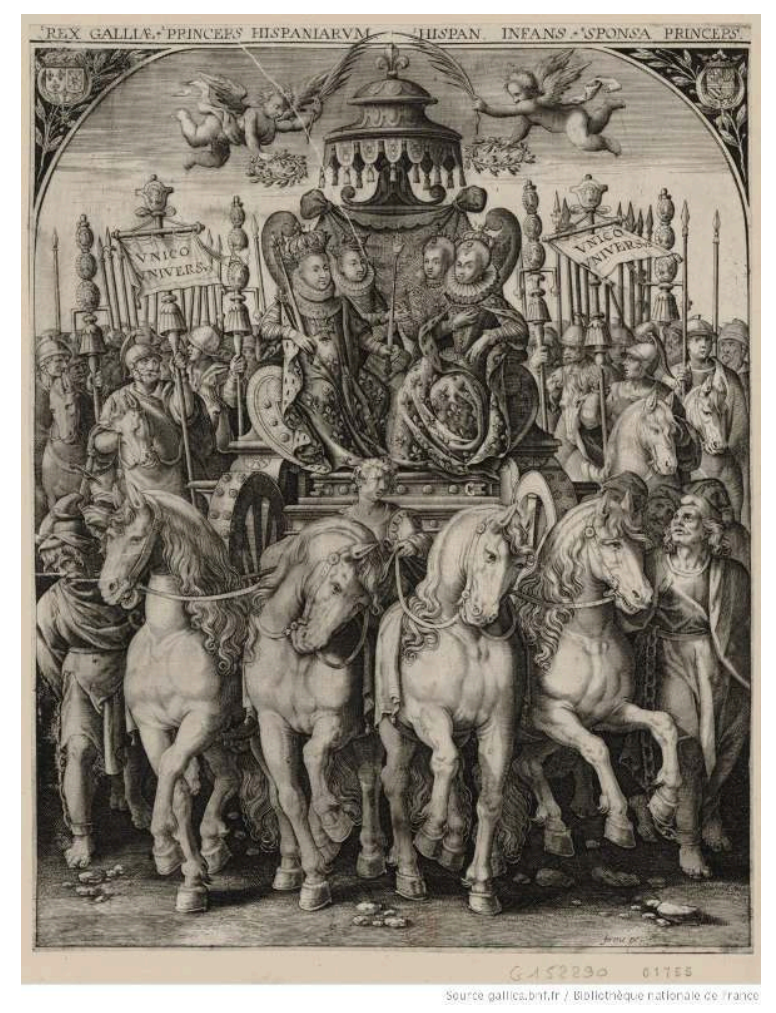

Pierre Firens (éditeur), Les Mariages franco-espagnols, 1612-1615, gravure, Paris, Bibliothèque nationale de France, collection Hennin $n^{\circ} 1755$.

(c) Bnf (c) Gallica

$13 \mathrm{Au}$ cours des vingt années pendant lesquelles Anne d'Autriche est reine régnante, une grande partie de ses représentations est conçue comme élément d'un diptyque dont le portrait du roi formerait l'autre face. Sur les pendants qui complètent l'image de la reine revêtue de son habit de majesté, Louis XIII apparaît le plus souvent revêtu de son armure. On l'observe aussi bien parmi les œuvres réalisées dans le cadre de la cour et qui s'adressent à un public restreint - ainsi le portrait de couple en pendant peint par 
Rubens durant les années où il travaille au cycle de la vie de Marie de Médicis ${ }^{32}$ - que dans le cadre d'une production gravée destinée à un public beaucoup plus large. Almanachs, petites planches gravées diffusées en feuilles ou illustrations de livres contribuent ainsi à la diffusion du motif et à son intégration à une culture visuelle partagée de la royauté.

Le frontispice d'un recueil de portrait des rois et reines de France, une des productions très populaires imprimées chez les éditeurs de la rue Montorgueil, en offre un exemple $^{33}$. Un autre frontispice réalisé juste après le mariage de 1615 permet de préciser le rapport qui se créé entre chacun des époux et les attributs guerriers représentés ${ }^{34}$ : le roi y est juché sur des trophées d'armes, tandis que la reine, qui tient un arc et une flèche, se tient sur des arcs et des carquois. Le texte du livret insiste sur la symbolique amoureuse de ces objets qui renvoie aussi bien à la chasse qu'aux attributs du dieu Cupidon, lequel figure d'ailleurs au registre inférieur. Ainsi, si la force du roi s'exerce sur le terrain militaire, la reine est elle-même dépositaire d'une autre force qui est tout aussi essentielle sur le plan politique : l'amour qui est le moteur de la fécondité grâce à laquelle la dynastie pourra assurer sa perpétuation.

La conception duale de la puissance armée de la royauté qui prend forme dans ces images du couple royal est donc conçue sous le régime de la complémentarité. Dans une grande planche gravée par Cornelis Van Dalen ${ }^{35}$, l'artiste d'Amsterdam a repris la composition d'une estampe gravée deux ans plus tôt par Robert Van Voerst, qui offrait la traduction d'une peinture du couple royal anglais réalisé en 1632 par Anton Van Dyck. Sur l'œuvre originale, le roi Charles Ir offrait un rameau d'olivier, symbole de la paix, à la reine Henriette-Marie, tandis que celle-ci présentait au roi une couronne de laurier, saluant ses victoires militaires. Dans la version "francisée ", où même le paysage est remplacé par une vue de Paris, le rameau d'olivier a disparu, et Louis XIII, à la différence de Charles $I^{\text {er }}$, est vêtu d'une armure, tandis qu'apparaît derrière lui un casque qui est encore plus visible que la couronne royale. Cette insistance sur l'iconographie du "roi de guerre" n'escamote toutefois pas la reine qui semble ici jouer le rôle d'une allégorie de la victoire, comme si sa dignité lui assurait une participation symbolique à la gloire militaire du souverain, bien qu'elle ne prenne pas part à la violence des combats. 
Fig. 4

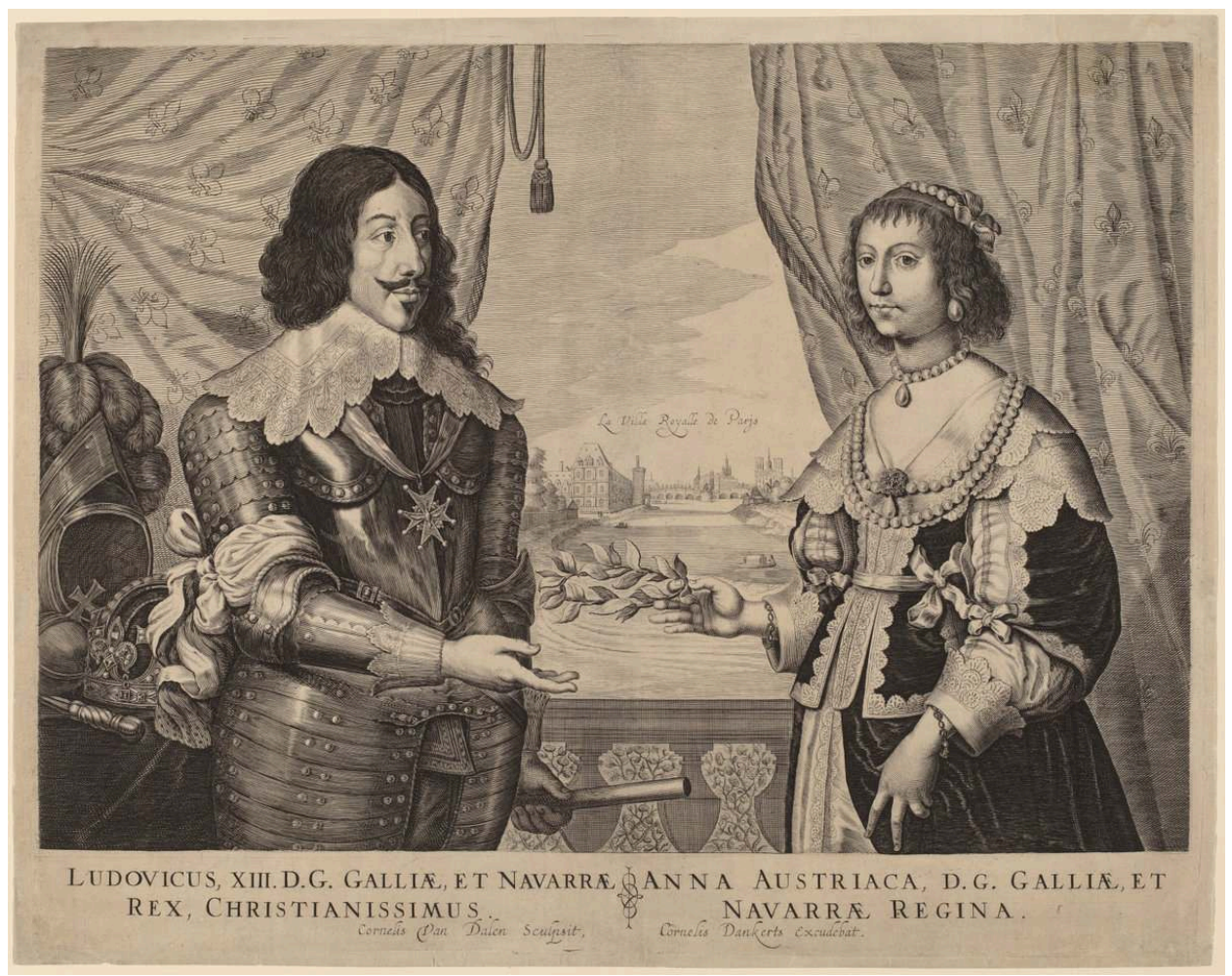

Cornelis Van Dalen, Louis XIII et Anne d'Autriche, vers 1635, gravure, Washington, National Gallery of Art, Rosenwald Collection.

(C) Washington, National Gallery of Art

Cette participation avait été renouvelée dans les dernières années du règne de Louis XIII, au moment où la reine, longtemps fragilisée à la cour par l'absence d'héritier, avait finalement connu un retour en grâce après la naissance de deux fils, Louis en 1638 et Philippe en 1640. Parmi les planches qui offrirent une diffusion visuelle à la famille royale, on vit ainsi réapparaitre le thème de l'ambition impériale et du triomphe romain, rappelant les œuvres produites trente ans plus tôt ${ }^{36}$.

17 À la différence de l'appropriation opérée par Marie de Médicis dans le Triomphe de Juliers, où le jeune Louis XIII n'est même pas représenté alors que c'est en son nom que la victoire est gagnée, les images d'Anne d'Autriche reposent toutefois sur une distinction nette entre le roi et la reine. Une estampe gravée pour célébrer des victoires de Louis XIII en 1642 est ainsi construite sur une stricte symétrie, présentant le monde de la guerre à gauche, tandis qu'à droite, derrière une personnification de la France, la reine se tient dans un palais, image de la protection qu'elle assure, loin du front, aux héritiers de la couronne ${ }^{37}$. Juste après la mort de Louis XIII, cette estampe populaire est retravaillée ${ }^{38}$, en effaçant la référence aux victoires précises de Collioure et de Perpignan pour devenir une image générique de la transmission de l'autorité, qui rappelle la composition d'une peinture essentielle de la galerie de Marie de Médicis par Rubens, La Remise de la régence, où se faisaient face le monde de la guerre et la continuité du gouvernement assuré par une femme et rendu au nom de l'héritier. 
Fig. 5

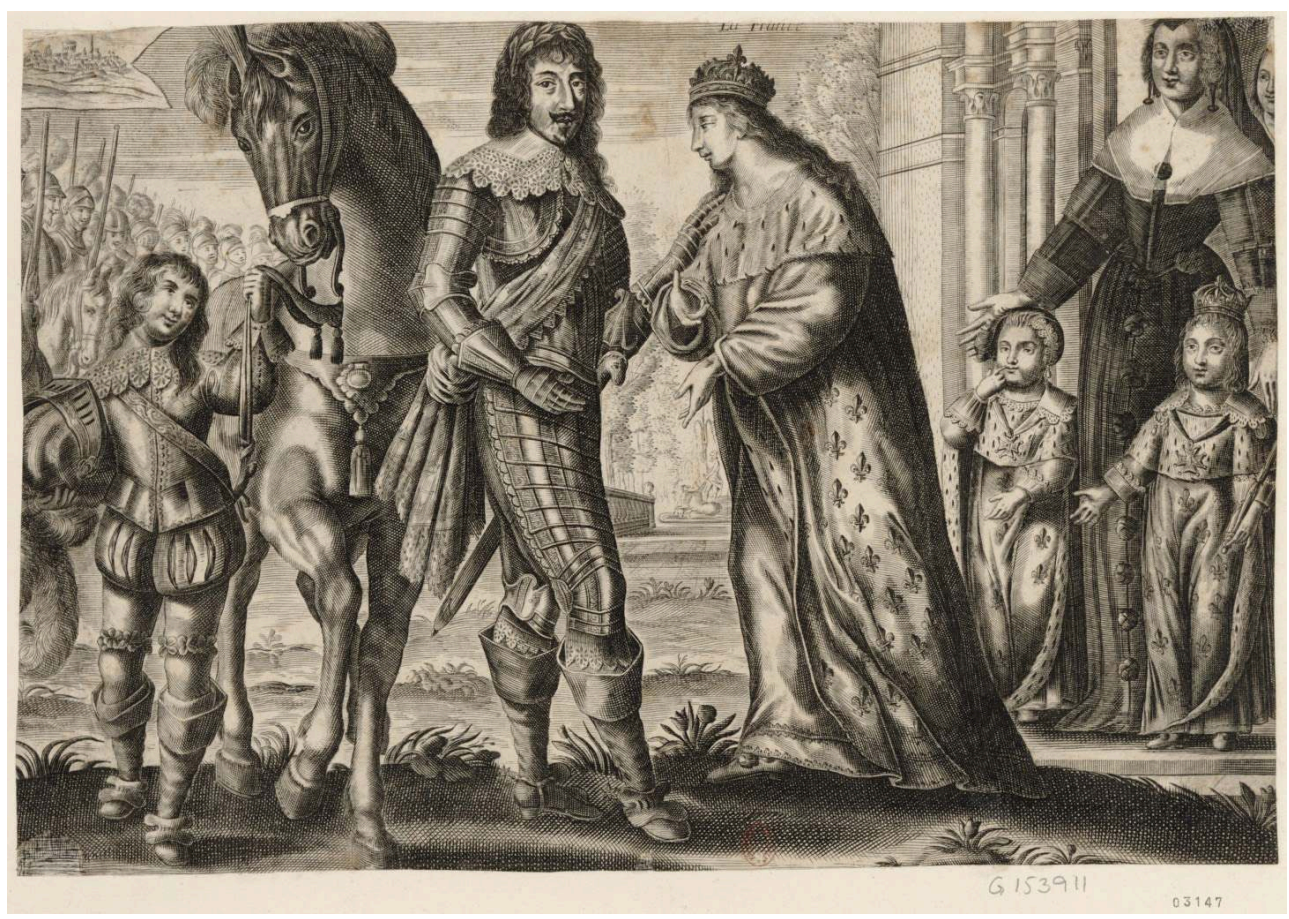

Anonyme, La France rendant hommage à Louis XIII, vers 1643, gravure, Paris, Bibliothèque nationale de France, collection Hennin $n^{\circ} 3147$

(c) Bnf (c) Gallica

Les images nombreuses qui sont répandues et diffusées pour présenter chaque victoire militaire obtenue durant la régence d'Anne d'Autriche ne cherchent ainsi jamais à effacer le jeune roi $^{39}$. Si la reine est toujours présente, et parfois même guide de la main le geste du souverain, c'est bien toujours Louis XIV qui revendique les attributs du pouvoir, que la couronne soit sur sa tête ou à ses côtés. La reine participe bien à la victoire militaire, mais au nom d'une délégation de pouvoir, un mandat de régence temporaire qui renvoie, in fine, au jeune roi comme seul dépositaire légitime de l'autorité royale.

Ce discours diffusé dans l'imagerie populaire et au sein de sphères de public assez larges est aussi celui, plus officiel, qui s'exprime dans les œuvres réalisées dans l'environnement de la cour. Sébastien de Beaulieu, ingénieur militaire au service de Louis XIII puis du jeune Louis XIV, répond ainsi à une commande royale en produisant en 1655 une immense carte murale représentant le siège victorieux d'Arras l'année précédente ${ }^{40}$. Cette entreprise manifeste de glorification royale était en outre ornée de sept médaillons, présentant, au registre inférieur, les acteurs historiques de la bataille, Mazarin et les maréchaux Turenne, la Ferté Seneterre et d'Hoquincourt, tandis qu'en partie supérieure Louis XIV occupait le médaillon central, entouré d'Anne d'Autriche à gauche et de Philippe d'Anjou à droite.

La présence de la reine mère sur un tel plan militaire est exceptionnelle. Les portraits de ces personnages sur le plan ne sont pourtant pas de simples hommages rendus à la famille royale, mais constituent une référence directe à leur présence, non pas au siège, mais dans les jours suivants, lorsque Louis XIV avait fait son entrée solennelle dans la ville. Le portrait de la reine nourrissait en outre plusieurs rapports avec le récit historique, puisque la ville d'Arras avait déjà été conquise en 1640, sous Louis XIII, 
victoire qui avait été, à l'époque, d'autant plus célébrée comme un signe divin qu'elle était survenue le jour de la saint Laurent - revanche symbolique sur la défaite française devant les armées espagnoles à la bataille de Saint-Quentin en 1557 - et alors que la reine était enceinte de son deuxième fils. L'image, aux dimensions à la fois historique, géographique et symbolique, tissait ainsi un réseau de significations serré entre la dignité royale, l'identité dynastique et l'affirmation de la force guerrière, où la reine prenait toute sa place - d'autant plus que dans cette représentation, à la différence des estampes populaires, la reine apparaissait non plus sous ses vêtements historiques son manteau noir et son voile de veuve - mais dans l'apparat intemporel de l'habit de majesté.

\section{Conclusion}

Les deux reines et régentes de France du premier XVIIe siècle entretiennent donc des rapports différents au thème de la violence visuelle dans le corpus de leurs représentations. Dans le portrait mythologique, Marie de Médicis incorpore l'esprit guerrier et s'approprie les armes - instruments et métaphores de la contrainte physique sur laquelle s'appuie le pouvoir royal-pour répondre aux enjeux contemporains et personnels : défendre sa présence, toujours fragile, au plus près $d u$ trône. L'échec final de ses prétentions en fait néanmoins un contre-modèle aux yeux d'Anne d'Autriche. Celle-ci conserve sa place sur le champ de bataille, et en démultiplie l'impact par l'usage des supports de grande diffusion, mais elle modifie les modalités de sa participation, délaissant la valeur guerrière, triomphale et violente de la figure de Minerve, telle qu'on la trouvait utilisée à la même époque chez les Frondeuses ${ }^{41}$. Chez la reine, la déesse devient tout au contraire, dans les dernières années de son existence, l'affirmation paisible d'une Paix, dont elle semble transmettre la mission - symbolique et politique - à sa belle-fille, Marie-Thérèse, dans un portrait très officiel présenté à la jeune Académie royale de Peinture et de sculpture ${ }^{42}$.

\section{NOTES}

1.

Denis Crouzet, Les guerriers de Dieu. La violence au temps des troubles de religion, vers 1525-vers 1610, Seyssel, Champ Vallon, 2005. Dans le champ des images, le caractère ambivalent d'une iconographie du roi violent avait été exploité aux dépens de Charles IX et d'Henri III. Voir Yann Lignereux, Les rois imaginaires. Une histoire visuelle de la monarchie de Charles VIII à Louis XIV, Rennes, PUR, 2016, pp. 100-106.

2.

Joël Cornette, Le Roi de guerre. Essai sur la souveraineté dans la France du Grand siècle, Paris, Payot, 1993. Pour un cadre spatial et chronologique plus large, voir Margaret M. McGowan, Festival and Violence. Princely Entries in the Context of War, 1480-1635, Turnhout, Brepols, 2019.

3. 
Une version de ce tableau est conservée au musée national du château de Pau, inv. P. 84.14.1. Il est consultable en ligne: https://www.pop.culture.gouv.fr/notice/joconde/00000060292 [site consulté le 23/07/2019]. Voir aussi cat. d'exp. Marie de Médicis : un gouvernement par les arts, sous la direction de Paola Pacht Bassani, Thierry Crépin-Leblond, Nicolas Sainte Fare Garnot et Francesco Solinas, Blois, 29 novembre 2003-28 mars 2004, Paris, Somogy / Blois: Château de Blois, 2003, p. 158.

4.

Sur une représentation gravée de la réduction de la ville de Perpignan (1642), Louis XIII apparaît ainsi à cheval au milieu de la mêlée des soldats (BnF, coll. Hennin, n3146, https://gallica.bnf.fr/ ark :/12148/btv1b84037969 [site consulté le 23/07/2020]). Voir Olivier Bonfait, « Quel héros pour le Roi ? Monarchie et héroïsation d'Henri IV à Louis XIV », Ralf von den Hoff, Felix Heinzer, Hans W. Hubert et Anna Schreurs-Morét (dir.), Imitatio heroica. Heldenangleichung im Bildnis, Würzburg, Ergon Verlag, 2015, p. 177, fig. 2. Quoiqu'il ne fût pas resté jusqu'à la capitulation définitive de la ville, Louis XIII avait bien été présent au siège.

5.

La métaphore, exploitée chez Henri IV comme une image de la lutte pour le retour de l'harmonie, réapparaît sous Louis XIII où elle met en valeur les victoires du roi guerrier. Voir Y. Lignereux, op. cit. note 1, pp. 116-117 et pp. 186-192.

6.

Max Weber, Le Savant et le politique, Paris, Plon, 1959.

7.

Norbert Elias, La Civilisation des mœurs, Paris, Calmann-Lévy, 1973.

8.

Robert Muchembled, Une histoire de la violence, de la fin du Moyen Âge à nos jours, Paris, Éditions du Seuil, 2008; Michel Nassiet, La violence, une histoire sociale. France, XVI ${ }^{e}$-XVIII ${ }^{e}$ siècles, Seyssel, Champ Vallon, 2011. Voir également les articles publiés dans Antoine Follain (dir.), Brutes ou braves gens ? La violence et sa mesure (XVI ${ }^{e}$ XVIII ${ }^{e}$ siècle), Strasbourg, Presses universitaires de Strasbourg, 2015.

9.

Voir essentiellement Fanny Cosandey, La reine de France. Symbole et pouvoir, XV ${ }^{e}$-XVIII ${ }^{e}$ siècle, Paris, Gallimard, 2000.

10.

Par exemple sur l'estampe anonyme La Vraye femme, vers 1650 (BnF, coll. Hennin, n3214, https:// gallica.bnf.fr/ark :/12148/btv1b84038601 [site consulté le 23/07/2020]).

11.

Abraham Bosse, La femme qui bat son mari (BnF, coll. Hennin, n2535, https://gallica.bnf.fr/ark :/ 12148/btv1b8403202h [site consulté le 23/07/2020]).

12.

Le thème visuel de la virago traduit un topos culturel misogyne qui parcourt les sociétés européennes depuis la fin du Moyen Âge ; voir notamment Sara F. Matthews-Grieco, Ange ou diablesse. La représentation de la femme au XVI e siècle, Paris, Flammarion, 1991, pp. 330-339 et Christa Grössinger, Picturing Women in Late Medieval and Renaissance Art, Manchester, Manchester University Press, 1997, pp. 112-124.

13.

Nicole Dufournaud, «Femmes en armes au XVI ${ }^{\mathrm{e}}$ siècle », Coline Cardi et Geneviève Pruvost (dir.), Penser la violence des femmes, Paris, La Découverte, 2012, pp. 343-349.

14.

Cette question a fait l'objet de nombreux travaux depuis une cinquantaine d'année ; voir surtout Ian MacLean, Woman Triumphant: Feminism in French Literature, 1610-1652, Oxford, Clarendon Press, 
1977 ; Mary D. Garrard, Artemisia Gentileschi: the Image of the Female Hero in Italian Baroque Art, Princeton, Princeton University Press, 1989 ; cat. d'exp. Die Galerie der Starken Frauen : Die Heldin in der französischen und italienischen Kunst des 17. Jahrhunderts, sous la direction de Bettina Baumgärtel et Silvia Neysters, Kunstmuseum Düsseldorf, 10 septembre-12 novembre 1995, Düsseldorf, Klinkhardt \& Biermann, 1995 ; et tout récemment Gilbert Schrenck, Anne-Élisabeth Spica et Pascale Thouvenin (dir.), Héroïsme féminin et femmes illustres (XVI ${ }^{e}$-XVII ${ }^{e}$ siècles). Une représentation sans fiction, Paris, Classiques Garnier, 2019. Sophie Vergnes offre un aperçu historiographique du sujet dans sa thèse de doctorat Les Frondeuses : l'activité politique des femmes de l'aristocratie et ses représentations de 1643 à 1661, Université Toulouse II - le Mirail, 2012 (https:// tel.archives-ouvertes.fr/tel-00760092 [site consulté le 23/07/2020]), pp.96-97. Sur la nature ambiguë de la participation du thème des femmes fortes à la «querelle des femmes ", voir enfin Marika Takanishi Knowles, "Tricky, Fine and Trapped: Painting the Femme Forte in Early Seventeenth-Century France ", Zeitschrift für Kunstgeschichte, 82-1, 2019, pp. 92-114.

15.

Selon les termes classiques des fonctions du portrait définies dans Louis Marin, Le Portrait du roi, Paris, Éditions de Minuit, 1981, p. 10.

16.

P. Pacht Bassani, T. Crépin-Leblond, N. Sainte Fare Garnot et F. Solinas (dir.), op. cit. note 3, p. 181.

17.

Catherine Pascal, «Représenter la régence? Image(s) de reine(s) dans les Éloges des douze dames illustres grecques, romaines et françoises dépeintes dans l'alcove de la reine (1646) », Sylvie Steinberg et Jean-Claude Arnould (dir.), Les Femmes et l'écriture de l'histoire, 1400-1800, Mont-Saint-Aignan, Publications des universités de Rouen et du Havre, 2008, pp. 89-102.

18.

Parmi l'abondante bibliographie sur ce décor, voir notamment deux contributions récentes: Nicolas Milovanovic, Du Louvre à Versailles. Lecture des grands décors monarchiques, Paris, Les Belles Lettres, 2005 et Elisabeth Oy-Marra, «Mazarin et les fresques de Giovanni Francesco Romanelli dans l'appartement d'été d'Anne d'Autriche au Louvre », Isabelle de Conihout et Patrick Michel (dir.), Mazarin. Les lettres et les arts, Paris, Bibliothèque Mazarine / Saint-Rémy-en-l'Eau, Éditions Monelle Hayot, 2006, pp. 144-155.

19.

Jean-Marie de Vernon, L'Amazone chrestienne, ou les avantures de Madame de S. Balmon, qui a conjoint heureusement durant nos jours une admirable devotion, \& la pratique de toutes les vertus, avec l'exercice des armes et de la guerre, Paris, Gaspar Méturas, 1678, p. 72. Malgré le témoignage de l'auteur, qui loue l'humilité de Madame de Saint-Balmont qui «fuyoit l'ostentation » et dont le portrait aurait été envoyé non par elle-même mais par ses amis, on peut douter que l'aristocrate n'ait pas été impliquée dans la diffusion assez importante de son image chez ses contemporains - deux portraits équestres du modèle par Claude Deruet sont encore aujourd'hui conservés, l'un au musée lorrain de Nancy (https://www.musee-lorrain.nancy.fr/fr/collections/les-oeuvresmajeures/portrait-equestre-de-madame-de-saint-baslemont-59 [site consulté le 23/07/2020]) et l'autre au musée Carnavalet (https://www.parismuseescollections.paris.fr/fr/musee-carnavalet/ oeuvres/portrait-equestre-d-alberte-barbe-d-ernecourt-dame-de-saint-balmont-1607\#infosprincipales [site consulté le 23/07/2020]). Sur le personnage, voir Micheline Cuénin, La dernière des Amazones, madame de Saint-Baslemont, Nancy, Presses universitaires de Nancy, 1992.

20.

Sur la question du portrait mythologique, voir principalement Françoise Bardon, Le Portrait mythologique à la cour de France sous Henri IV et Louis XIII. Mythologie et politique, Paris, A. et J. Picard, 1974.

21. 
Fernand Mazerolle, Les médailleurs français du XVe siècle au milieu du XVII siècle, Paris, Imprimerie nationale, 1902, tome II, pp. 128-129 ; Ronald Forsyth Millen et Robert Erich Wolf, Heroic Deeds and Mystic Figures. A New Reading of Rubens' Life of Maria de' Medici, Princeton, Princeton University Press, 1989, p. 101.

22.

Voir Jean-François Dubost, Marie de Médicis : la reine dévoilée, Paris, Payot, 2009.

23.

Parmi l'abondante bibliographie, voir notamment Jacques Thuillier et Jacques Foucart, Rubens, la Galerie Médicis au Palais du Luxembourg, Paris, R. Laffont / Milan, Rizzoli, 1969 ; R. Forsyth Millen et R. E. Wolf, op. cit. note 22 ; Fanny Cosandey, « Représenter une reine de France. Marie de Médicis et le cycle de Rubens au palais du Luxembourg ", CLIO. Histoire, femmes et sociétés, $\mathrm{n}^{\circ} 19$, avril 2004, pp. 63-83.

24.

«Description des tableaux de la gallerie du palais de la Royne mère du Roy, aux faulxbourgs Saint-Germain-lez-Paris », BnF, département des manuscrits, Baluze 323, f. 54 (https:// gallica.bnf.fr/ark:/12148/btv1b9001514c/f98.image [site consulté le 23/07/2020]); sur ce manuscrit, voir J. Thuillier et J. Foucart, op. cit. note 23 .

25.

Marianne Cojannot-Le Blanc et Évelyne Prioux, Rubens. Des camées antiques à la galerie Médicis, Paris, Le Passage, 2018, p. 54.

26.

R. F. Millen et R. E. Wolf, op. cit. note 23, pp. 224-225.

27.

Jean-François Dubost, «Henri IV au Pont-Neuf. Genèse, hésitations sémantiques et détournements d'une effigie royale (1604-1640)», Henri IV. Art et pouvoir, Rennes, PUR, Tours, PUFR, 2016, pp. 129-159.

28.

En s'appuyant sur l'esquisse conservée à l'Alte Pinakothek de Munich où c'est le roi qui paraît figurer en personne, Marianne Cojannot-Le Blanc va jusqu'à parler de «substitution », voir M. Cojannot-Le Blanc et É. Prioux, op. cit. note 25, p. 49.

29.

Sara Galletti, «Rubens's Life of Maria de' Medici : Dissimulation and the Politics of Art in Early Seventeenth-Century France », Renaissance Quarterly, n 3, automne 2014, pp. 878-916.

30.

Louis XIII, Anne d'Autriche, Philippe d'Autriche et Élisabeth de France assis dans un char traîné par quatre chevaux, estampe anonyme éditée par Pierre Firens (BnF, coll. Hennin, $\mathrm{n}^{\circ} 1755$, https:// gallica.bnf.fr/ark :/12148/btv1b8401700s [site consulté le 23/07/2020]).

31.

Voir notamment le camée du triomphe d'un empereur (peut-être Licinius). Paris, BnF, cabinet des médailles, reproduit dans M. Cojannot-Le Blanc et É. Prioux, op. cit. note 25.

32.

Pasadena, Simon Norton Foundation.

33.

Paris, BnF, coll. Hennin, $n^{\circ}$ 1570, https://gallica.bnf.fr/ark :/12148/btv1b84015359 [site consulté le 23/07/2020].

34.

Paris, BnF, coll. Hennin, $n^{\circ}$ 1751, https://gallica.bnf.fr/ark :/12148/btv1b8401696b [site consulté le 23/07/2020].

35. 
Paris, BnF, série N3 (portrait de Louis XIII).

36.

Voir par exemple l'estampe de Jaspar Isac, L'Æil de Dieu regarde la France, vers 1642 (Paris, BnF, série $Q b 1,1642$ ).

37.

L'Heureux retour du Roy Louis tres chrestien après avoir réduit à son obéissance les villes de Collioure et Perpignan (Paris, BnF, série Qb1, 1642).

38.

La France rendant hommage à Louis XIII, vers 1643 (Paris, BnF, coll. Hennin, $\mathrm{n}^{\circ} 3147$, https:// gallica.bnf.fr/ark :/12148/btv1b8403797q [site consulté le 23/07/2020]).

39.

Barbara Gaehtgens, "Prints, Politics, and a Child King: The Battle of Rocroi in 1643 », Getty Research Journal, no 7, 2015, pp. 1-18.

40.

Véronique Meyer, "Sébastien Pontault de Beaulieu et la levée du siège d'Arras ", Nouvelles de l'estampe, $\mathrm{n}^{\circ} 178$, novembre 2001, pp. 6-23.

41.

Paris, BnF, série Qb1, vers 1652, Le compliment de Mademoiselle en la ville d'Orléans aux Mazarins.

42.

Simon Renard de Saint-André, Anne d'Autriche et Marie-Thérèse d'Autriche, Versailles, musée du château, inv. MV 6925 (http://collections.chateauversailles.fr/ \#38d35bd7-4dfe-4828-9fdf-535c52df1746 [site consulté le 23/07/2020]).

\section{RÉSUMÉS}

Dans sa dimension guerrière, la violence devient l'un des attributs iconographiques fondamentaux de la royauté en France au XVII ${ }^{e}$ siècle. Bien qu'elles soient a priori écartées de la gestion des affaires en raison de la loi salique, les reines Marie de Médicis et Anne d'Autriche cherchent néanmoins à endosser ce costume martial du chef de guerre dans leurs portraits, en particulier lorsqu'une régence de minorité les place à la tête du gouvernement. Pour cela, elles ne se réfèrent toutefois pas à une formule unique mais conçoivent chacune des stratégies personnelles, divergeant par les moyens, les objectifs et les résultats, en empruntant tour à tour au portrait mythologique ou à la représentation historique pour définir leur rôle dans la conduite des conflits, et in fine dans la maîtrise du pouvoir royal.

In 17th-century France, the warlike dimension of violence became one of the fundamental iconographic attributes of royalty. Although at the outset they were excluded from managing affairs of state on account of Salic law, the queens Marie de' Medici and Anne of Austria nonetheless sought to wear the costume of the chief of command in their portraits, in particular when they were placed at the head of the government in the position of regent. To achieve this, however, they did not employ a single formula but each developed personal strategies that varied in their means, objectives and results, borrowing in turn from mythological portraiture and historical representation to define their role in the management of conflicts, and ultimately of royal power. 


\section{INDEX}

\section{Index géographique : France}

Keywords : Portraiture of power, gender and power, royal iconography, queen, France, 17th century, violence, Marie de Medici, Anne of Austria

Mots-clés : Portrait du pouvoir, genre et pouvoir, iconographie royale, reine, France, XVIIe siècle, violence, Marie de Médicis, Anne d'Autriche

Index chronologique : XVIIe siècle

\section{AUTEUR}

\section{DAMIEN BRIL}

Damien Bril est actuellement chercheur post-doctorant à l'École du Louvre dans le programme de recherche Collecta. Il travaille sur les représentations du pouvoir, le genre et les cultures visuelles en France et en Europe à l'époque moderne. Ancien élève de l'École du Louvre, de l'université Paris IV Sorbonne et de l'université de Provence, il a soutenu en 2018 une thèse de doctorat en histoire de l'art à l'université de Bourgogne Franche-Comté sur l'iconographie d'Anne d'Autriche.

Damien Bril is currently a postdoctoral researcher at the École du Louvre in the Collecta research programme. He works on representations of power, gender and visual cultures in France and Europe during the modern era. A former student at the École du Louvre, the Université Paris IV Sorbonne and the Université de Provence, in 2018 he presented a doctoral dissertation in art history at the Université de Bourgogne Franche-Comté on the iconography of Anne of Austria. 